\title{
Cuarenta años de actividad editorial de la Asociación Mexicana de Cirugía General, 1974-2014
}

\author{
Forty years of editorial activity of the Mexican Association of General Surgery \\ (Asociación Mexicana de Cirugía General), 1974-2014
}

Guillermo León López, ${ }^{*}$ Luis Mauricio Hurtado López, ${ }^{* *}$ Abilene C Escamilla Ortiz***

\footnotetext{
Palabras clave:

Historia de la cirugía general en México, revista Cirujano General, boletín;

Tratado de cirugía general, 1.a y $2 . \mathrm{a}$ ediciones.

Key words: Historia de la Cirugía General en México, Cirujano General journal, bulletin; Tratado de cirugía general, 1 st and 2 nd
} editions.

* Exeditor de la revista Cirujano General (1991-2005).

** Exeditor de la revista Cirujano General (2005-2013).

*** Editora de la revista Cirujano General (2015-actualidad).

Recibido: 16/12/2015 Aceptado: 15/04/2016

\section{RESUMEN}

Objetivo: Destacar las obras que la Asociación Mexicana de Cirugía General (AMCG) ha patrocinado y publicado a través de 40 años (1974-2014) de su historia. Material y métodos: Se realizó una revisión sistemática de las publicaciones periódicas y libros que la asociación ha editado en este lapso. Resultados: Dos han sido las publicaciones periódicas: Cirujano General (1980) y Boletín informativo (1993), ambas impresas en papel. Posteriormente, tanto el Boletín (2009), como la revista (2010) migraron a versión electrónica; empero, Cirujano General continúa imprimiendo 200 ejemplares para distribución en escuelas o facultades de medicina que no cuenten con Internet, u hospitales en semejantes condiciones. En el año 2003, con el apoyo de la editorial mexicana El Manual Moderno, se publicó la obra Tratado de cirugía general, 1. ${ }^{\mathrm{a}}$ edición, con 191 capítulos en 1,607 páginas escritos por cirujanos generales de 15 estados del país y la Ciudad de México. Cinco años después (2008), se imprimió la segunda versión de la obra, con una extensión de1,581 páginas y 192 capítulos escritos por cirujanos generales de 19 estados del país. Durante los años 2011, 2012 y 2013, la AMCG editó tres volúmenes de una serie que se denominó Historia de la Cirugía General en la República Mexicana. En cada uno de los volúmenes se describió el inicio, desarrollo y estado actual de la cirugía general en 15 estados del país y en 10 hospitales de la Ciudad de México; todos los autores eran oriundos de esos estados. En 2012 y 2013, otras dos obras se publicaron bajo el sello de la AMCG: Historia de la Asociación Mexicana de Cirugía General y Perfiles: Cirujanos Generales Mexicanos del Siglo XX, con el apoyo de la editorial Graphimedic, SA de CV.

\section{ABSTRACT}

Objective: To highlight the works that the Mexican Association of General Surgery (AMCG) has sponsored and published in its history of 40 years (1974-2014). Material and methods: A systematic review was performed of the periodicals and books that the association has published during this period. Results: There have been two periodicals: Cirujano General (1980) and Boletín informativo (1993), both printed on paper. Later, both the bulletin (2009) and the journal (2010) migrated to electronic version; however, Cirujano General continues to print 200 copies for distribution in schools or medical schools that do not have Internet, or hospitals under similar conditions. In 2003, with the support of the Mexican publishing house El Manual Moderno, we published the first edition of the Tratado de cirugía general, with 1,607 pages in 191 chapters, written by general surgeons from 15 states and Mexico City. Five years later (2008), the second version was printed, with an extension of 1,581 pages and 192 chapters by general surgeons from 19 states. During the years 2011, 2012 and 2013, the AMCG edited three volumes of a series that was called Historia de la Cirugía General en la República Mexicana. Each of the volumes describes the birth, development and current state of general surgery in 15 states and 10 hospitals in Mexico City; the authors are all originally from those states. In 2012 and 2013, two other works were published by the AMCG: Historia de la Asociación Mexicana de Cirugía General and Perfiles: Cirujanos Generales Mexicanos del Siglo XX, with the support of the publishing house Graphimedic, SA de CV.
"Si los médicos no rescatamos aunque sean fragmentos de la historia de la cirugía en nuestro país, nadie lo va a hacer".

Dr. Ernesto de la Rocha de la Rocha. Cirujano General. Chihuahua, Chih.

\section{ANTECEDENTES}

$\mathrm{L}$ a asociación, en sus estatutos, ${ }^{1}$ en el artículo 2, describe el objeto de su ser: "La Asociación es una corporación no lucrativa que tiene por objeto: 1) El estudio de los problemas relacionados con la cirugía general y ramas afines; 
2) La investigación científica, el mejoramiento de la técnica, el progreso de dicha especialidad y el desarrollo de labores educativas; el mejoramiento y el progreso de sus miembros en los aspectos técnicos, legales, culturales, morales y éticos".

Citamos lo anterior porque algunos socios comentan que la asociación no debería consumir sus recursos económicos en la edición de libros que no son estrictamente científicos y que en caso de hacerlo, éstos deberían ser autofinanciables. Afortunadamente, los cirujanos que han conducido los destinos de nuestra corporación han cumplido a cabalidad con lo que el estatuto demanda: "no sólo técnica quirúrgica, no sólo ciencia, también cultura y humanismo".

Actualmente, nuestra organización académica está constituida por la Asociación Mexicana de Cirugía General (fundada oficialmente el 4 de agosto de 1973 y que cuenta con una membresía de 2,930 asociados) y la Federación Nacional de Colegios y Asociaciones de Especialistas en Cirugía General (fundada el 21 de febrero de 2006 y agrupa a 21 colegios).

\section{EL GERMEN DE LA IDEA Y LOS VISIONARIOS}

A manera de preámbulo, antes de hacer referencia a las publicaciones de la asociación, debemos citar lo escrito por uno de los fundadores de la misma, el Dr. Salvador Rodríguez Martínez, en el artículo "Algunos hechos no conocidos de la fundación de la AMCG". ${ }^{2}$ Escribió él:

"Las primeras ideas de su creación se gestaron en un pequeño café localizado frente a la puerta principal del Hospital General de la SSA; allí, el Dr. Felipe Zaldívar Bernal, en aquel tiempo jefe del Servicio de Cirugía General de ese hospital, manifestó al pequeño grupo de colegas reunidos con él la conveniencia de que los cirujanos generales mexicanos, dramáticamente huérfanos de un hogar científico, se agruparan en una sociedad científica que los acogiera a todos por igual." 2

Contrariamente a lo que ocurre en infinidad de charlas de café, estos cirujanos, hombres de

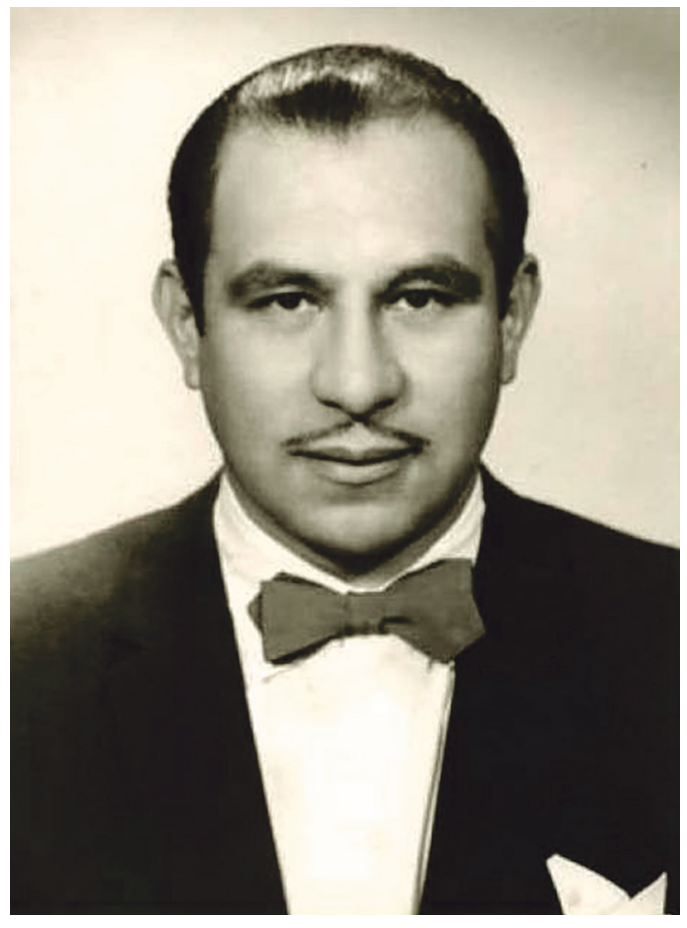

Figura 1. Dr. Felipe Zaldivar Bernal.

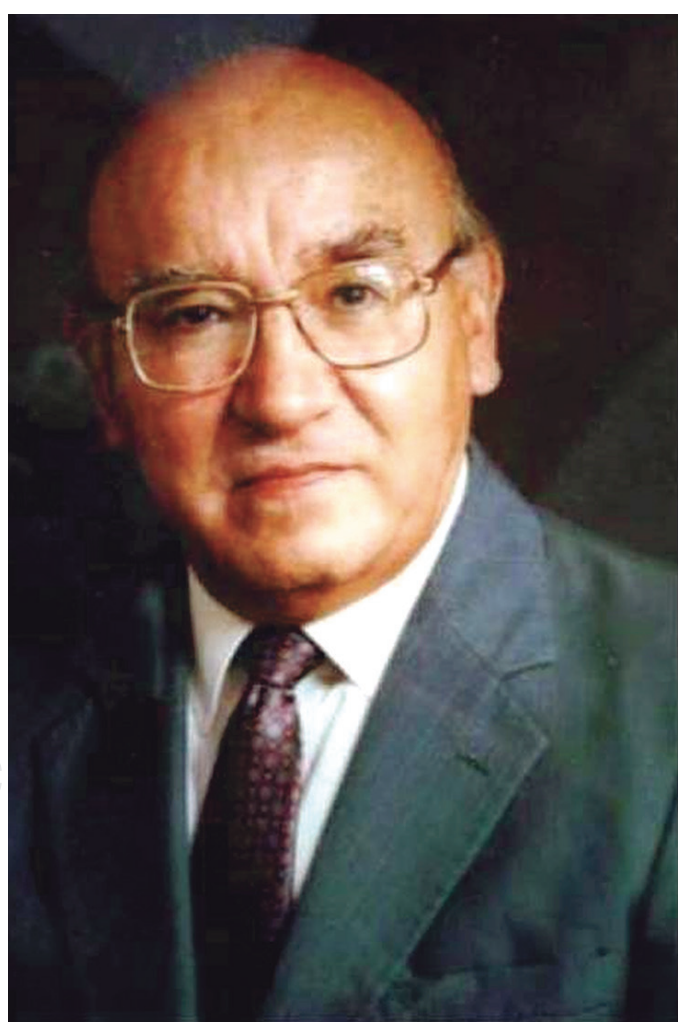

Figura 2. Dr. Salvador Rodríguez Martínez. 
acción al fin, se pusieron a trabajar en serio, y la primera acción que tomaron fue integrar un comité organizador en el que se distribuyeron los cargos de la siguiente forma: Felipe Zaldívar Bernal (presidente) (Figura 1), Rafael Díaz García Rojas (secretario), Salvador Rodríguez

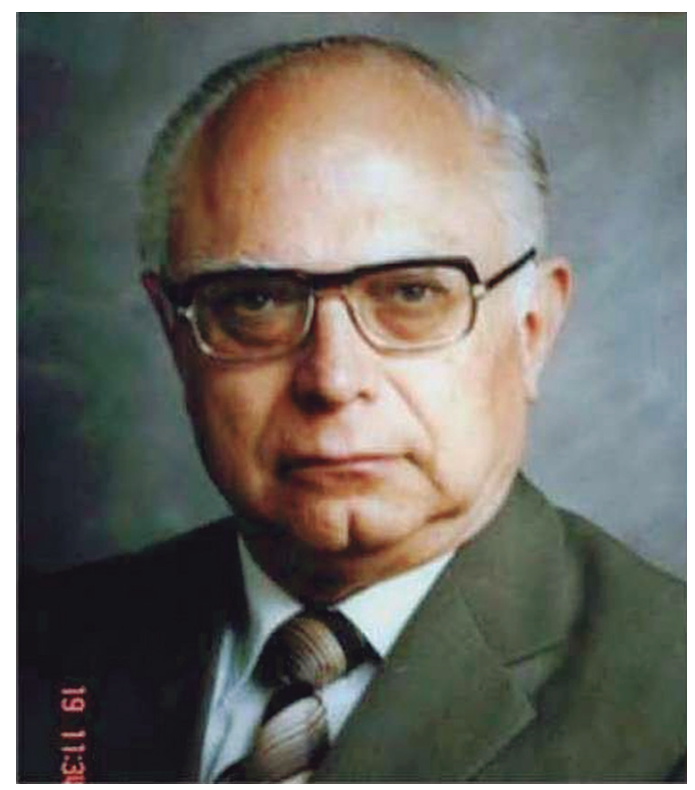

Figura 3. Dr. Enrique Fernández Hidalgo.

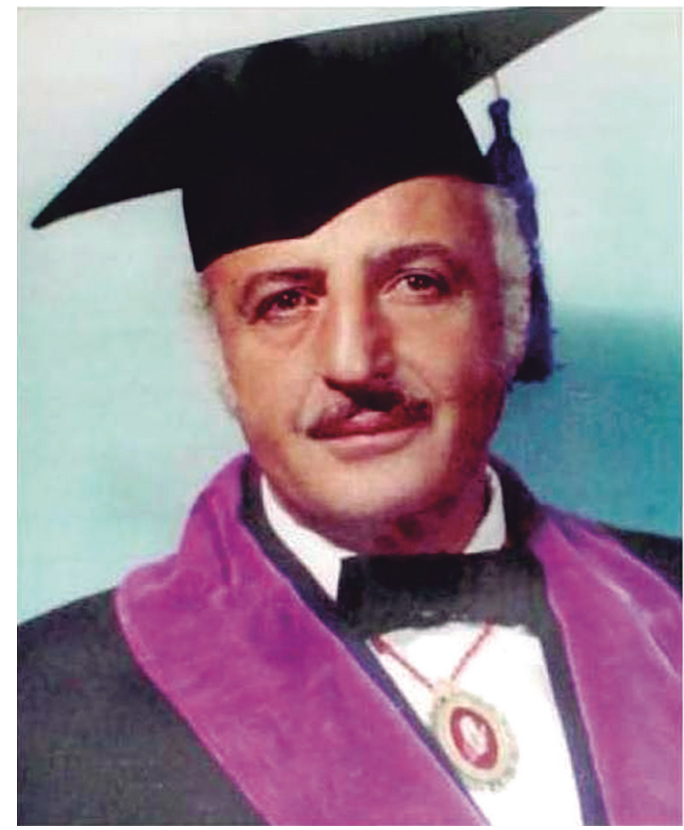

Figura 4. Dr. Roberto Haddad Slim.
Martínez (Figura 2) (tesorero), Enrique Fernández Hidalgo (Figura 3), primer vocal, y Roberto Haddad Slim (Figura 4), segundo vocal.

"El día 4 de agosto de 1973, —continuamos citando a Salvador Rodríguez Martínez- ante el Notario Público \#13 de la Cd. de México, DF, el comité organizador registró a la corporación como asociación civil con el nombre de Asociación Mexicana de Cirugía General". El 31 de octubre del mismo año, el autonombrado comité organizador convocó a una asamblea extraordinaria en punto de las 20 horas, - enfatiza nuestro cronista- que se realizó en el otro hospital emblemático de la cirugía en ese entonces, el Hospital Juárez de la SSA.

El asunto principal de la asamblea era elegir la primer mesa directiva de la naciente asociación; participaron en el debate 56 cirujanos. La primer mesa directiva elegida quedó conformada así: presidente: Dr. Octavio Montañez Martínez, vicepresidente: Dr. Felipe Zaldívar Bernal, secretario: Dr. Roberto Pérez García, tesorero: Dr. Salvador Rodríguez Martínez, y vocal: Dr. Enrique Fernández Hidalgo.

Como se aprecia, de los cinco integrantes del comité organizador, únicamente dos llegaron a ocupar la presidencia de la asociación. El por qué los tres restantes no, queda en el terreno de la suposición; quizás con el paso del tiempo se adhirieron a la organización cirujanos más jóvenes y mejor calificados, o quizá no les interesó hacerlo.

\section{CIRUJANO GENERAL}

Refiere Salvador Rodríguez Martínez, sobreviviente, junto con el Dr. Enrique Fernández Hidalgo, de aquel grupo de cinco cirujanos que conformaron el comité organizador, que en el estatuto primigenio de 1975, en el capítulo 14 , se indicaba que debería existir un órgano editorial oficial de la asociación, que se denominaría Revista de cirugía general de México. En 1980, durante la realización del 3. ${ }^{\text {er }}$ Congreso Nacional de Cirugía General, realizado en la ciudad de Guanajuato y organizado por el comité directivo del cual era presidente el propio Dr. Rodríguez Martínez, se entregó a los asociados, cinco años después de fundada la asociación, el primer número (Figura 5) de la susodicha revista. iPero el título de la revista 


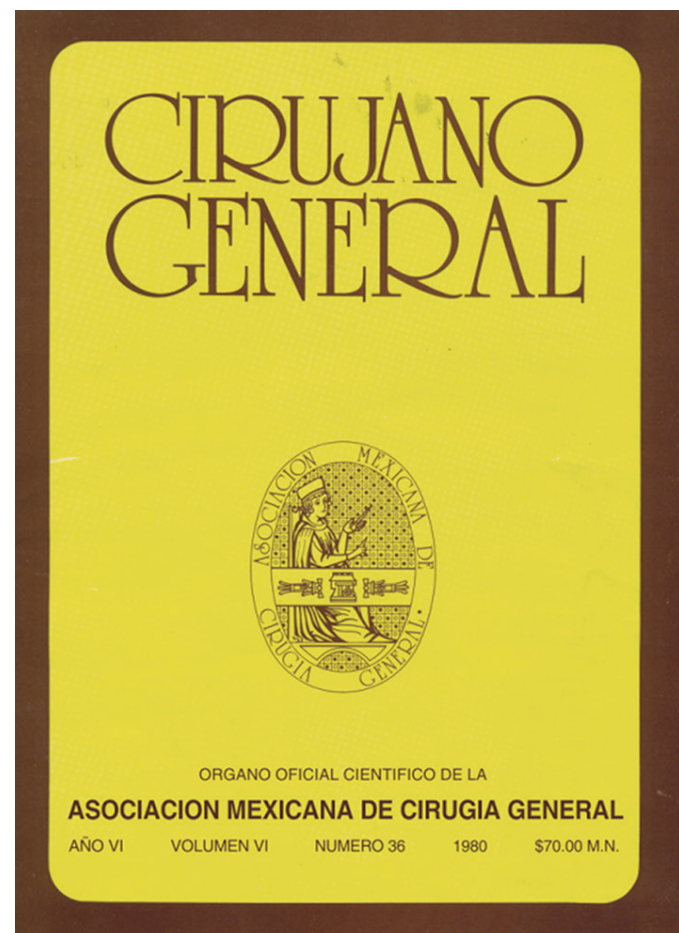

Figura 5. Primer número de la revista Cirujano General.

que se entregó no era Revista de Cirugía General de México, sino el actual: Cirujano General!

¿Por qué el cambio de nombre de la revista? No se especifica con certidumbre; el propio Dr. Rodríguez señala que hubo muchas discusiones en las reuniones del comité directivo que él presidía, y que finalmente se decidió el actual por su brevedad; eran sólo dos palabras, lo que hacía fácil recordarlo y nombrarlo, y evitaba repetir la manida frase de "Revista de.... de México", nombre extenso y que copiaba el de otras publicaciones. Finalmente, tal vez evocaba la brevedad del nombre de la revista de la Academia Mexicana de Cirugía: Cirugía y Cirujanos.

En el transcurso de 1990 encontramos casi todos los ejemplares sobrantes que se habían publicado hasta ese año e identificamos el que parecía ser el primero, cuyo número de catálogo era Año VI, Vol. 6; 1980, Núm. 36. Sin embargo, llamaron la atención dos hechos. El primero, que la mesa directiva que presidía la asociación en 1980 era encabezada por el Dr. Salvador Rodríguez Martínez (3. ${ }^{\text {er }}$ presidente), y en la hoja de créditos del núm. 36 aparece como presidente de la AMCG el Dr. Oscar Díaz Giménez, cuyo periodo fue durante el bienio 1981-1982 (4. ${ }^{\circ}$ presidente); en el libro Historia de la Asociación Mexicana de Cirugía General, ${ }^{3}$ el Dr. Díaz Giménez no se acredita el primer número de Cirujano General (páginas 43-46). El segundo hecho es que en ese mismo libro, el Dr. Salvador Rodríguez Martínez, tercer presidente de la AMCG, 1979-1980, refiere que "el primer número de Cirujano General fue entregado a los asistentes en la reunión anual efectuada en Guanajuato, celebrada del 25 al 27 de septiembre de 1980, cinco años después de fundada la AMCG" (página 42). Le solicitamos en préstamo el único ejemplar que tenía en su poder de ese primer número y lo envió desde la ciudad de Cholula, Puebla, donde reside actualmente. De él tomamos los datos de la portada, que correspondieron al primer número de Cirujano General, con la catalogación: Año VI, Vol. VI, Núm. 36 (Figura 5).

Al cuestionar a Salvador Rodríguez Martínez del por qué de esta decisión de iniciar la revista con el año VI, Vol. VI, Núm. 36, nos reveló el trasfondo de ello: "La naciente asociación carecía de liquidez; las cuotas aportadas por los 185 socios fundadores apenas si cubrían los gastos de papelería, el diploma para el socio, etcétera. La penuria era tal que los directivos sesionaban en el consultorio particular del presidente en turno; menos iban a ser suficientes para editar una revista". Para lograr esto, recurrieron a lo que hoy se conoce como "estrategia de mercado". Siguiendo esta idea, la mesa directiva en pleno solicitó asesoría a la empresa editorial Grupo Infocorp, S.A. de C.V., quien recomendó, para conseguir financiamiento, iniciar la catalogación de la publicación de la siguiente forma: Año VI, Vol. VI, número 36 (Figura 5).

Pero se pagó un costo, también de acuerdo con la lógica del mercado: el editor tuvo que aceptar que de las 29 páginas del primer número, 16 fueran para los artículos y 13 para publicidad; en el siguiente número, el 37, 18 páginas fueron para los artículos y 14 para publicidad; en el número 38, 10 y 11 ; y en el número 39, 11 y 11 , respectivamente. A partir del número 40, de 1981, los anuncios se confinaron a la segunda, tercera y cuarta de forros. Los editores subsecuentes corrigieron la catalogación. 


\section{CRUJANO GENERAL}

ORGANO OFICIAL CIENTIFICO DE LA

ASOCIACION MEXICANA DE CIRUGIA GENERAL

Año VIII VOLUMEN VII NUMERO 41982

Figura 6. Portada de la revista Cirujano General en 1982.

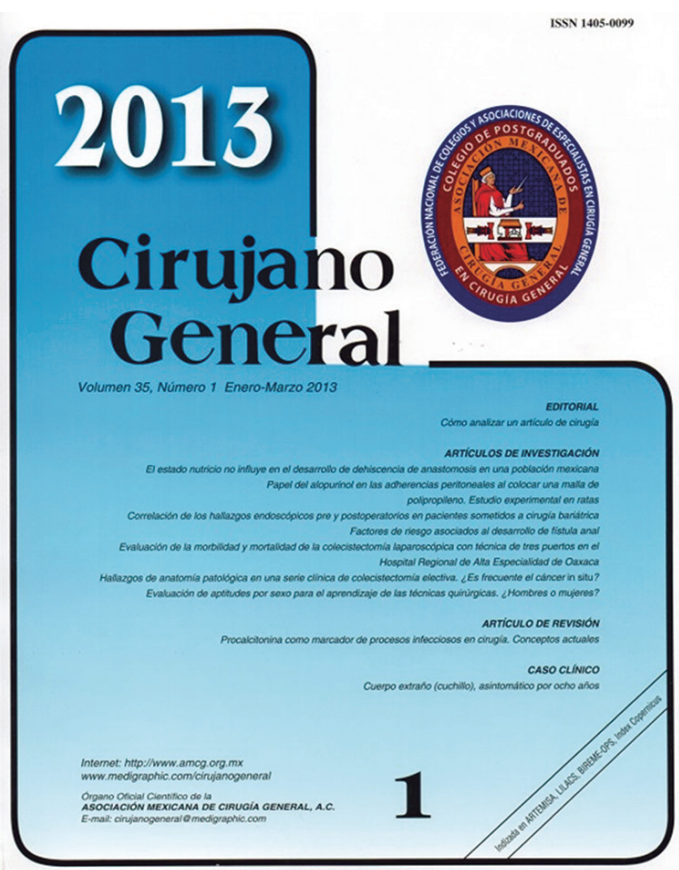

Figura 7. Portada de la revista Cirujano General en 1988 a 2013
Sin entrar en detalles, revisemos brevemente algunas de las portadas que ha tenido la revista durante sus 35 años de publicación ininterrumpida: en 1981, la portada cambió de amarilla a negra; en 1982 (Figura 6), se utilizó una portada de color azul; en 1988 se reestructuró la portada con un diseño diferente (Figura 7), con modificaciones menores; hasta el año 2013 y en 2014 cambió su formato (Figura 8), la portada ha tenido 12 modificaciones, siempre con la intención de adaptarse a los tiempos modernos.

El contenido del primer número se muestra en la figura 9; fueron en total seis artículos, con la participación de 18 autores que provenían de cinco instituciones hospitalarias del Distrito Federal y uno de Poza Rica, Veracruz.

\section{LOS EDITORES (FIGURA 10)}

Once han sido los cirujanos que han dirigido la publicación durante 35 años, todos ellos con el compromiso de editarla trimestralmente, sin retrasos ni errores, cumpliendo con la normatividad que señalan las instrucciones para las

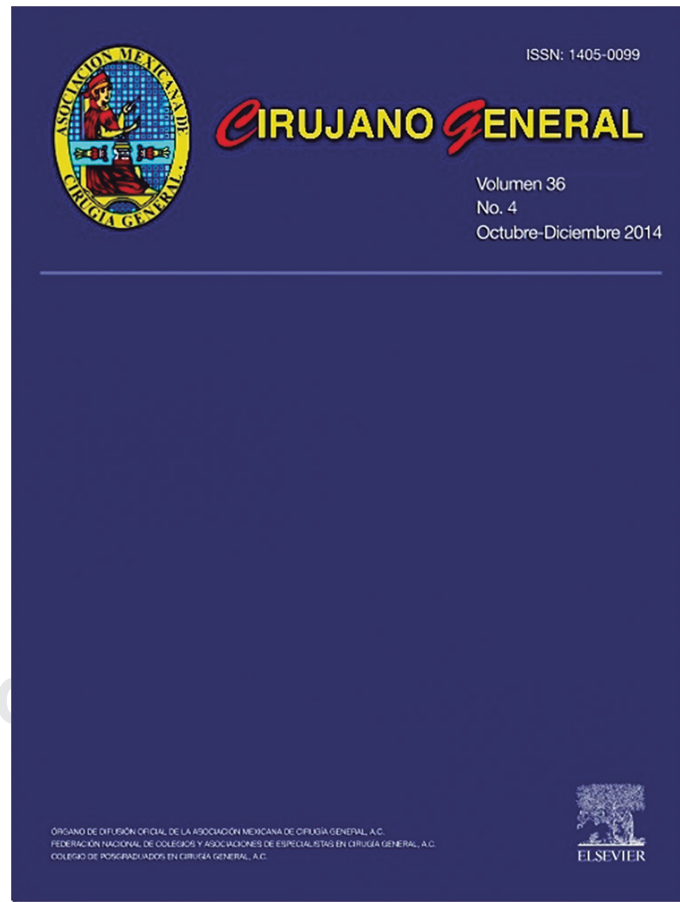

Figura 8. Portada de la revista Cirujano General de 2014. 
1. "Anatomía patológica del paciente con trasplante renal" Cinco autores. (Centro Hospitalario 20 de Noviembre. ISSSTE. DF)

2. "Hernia inguinal en pediatría" Tres autores. (Hospital Juárez, SSA (DF) y Hospital Infantil Inguarán. DF)

3. "Obstrucción intestinal aguda por cáncer colorrectal" Cuatro autores. (Hospital Juárez, SSA. DF)

4. "Anastomosis coledoco-duodenal" Tres autores. (Hospital Inglés ABC. DF)

5. "Hernioplastía umbilical" Dos autores. (Hospital General de zona \# 58 del IMSS, DF)

6. "Quiste pancreático" Un autor. (Hospital Regional de PEMEX. Poza Rica, Ver.)
Figura 9.

Contenido del primer número de Cirujano General.
1. 1980
2. 1981-1987
3. 1987
4. $1987-1990$
5. 1987-1990
6. 1990-2005
7. 2002-2005
8. 2005
9. 2006-2013
10. 2013-2014
11. 2015

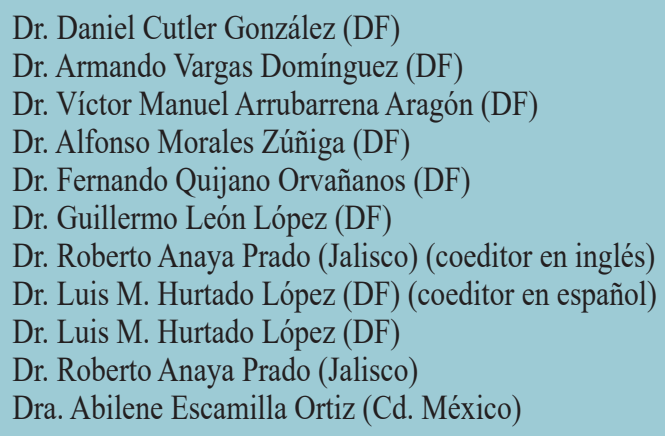

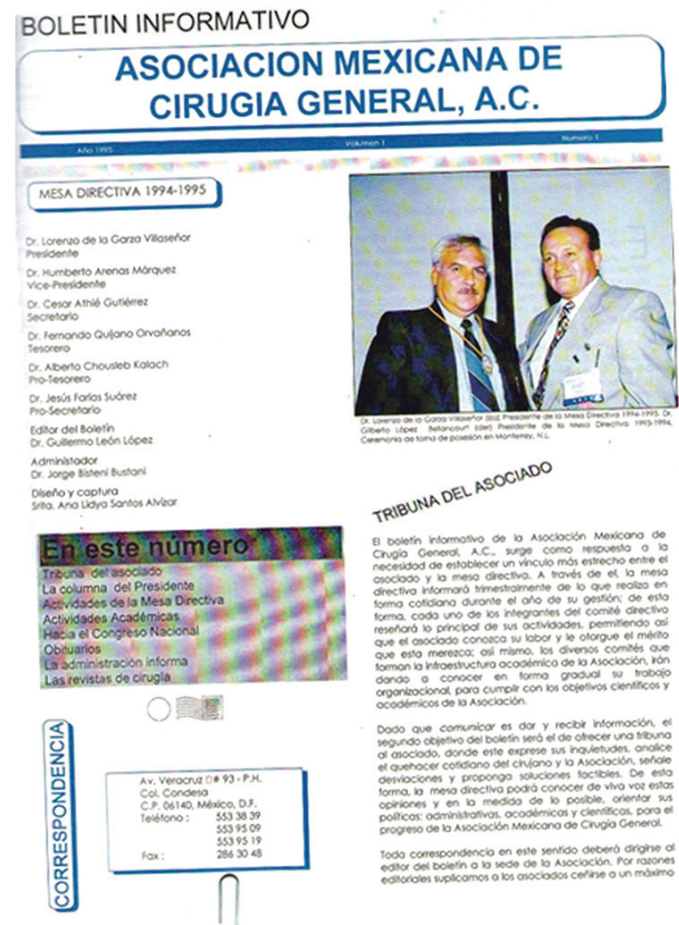

Figura 11. Boletín informativo. publicaciones periódicas. Asimismo, estimular a los asociados para publicar sus estudios clínico-quirúrgicos y de investigación e intentar incluirla en los principales índices de publicaciones periódicas nacionales e internacionales: Índice Bibliomex-Salud (UNAM-IMSS-SS), impreso de 1994 a 2002; Salud-Ciencia (Sociedad Iberoamericana de Información Científica), impreso de 1994 a la actualidad; portal Graphimedic-Cirujano General, electrónico, de 2001 a la actualidad; Google Académico, electrónico, de 2001 a la actualidad; Periódica, Índice de Revistas Latinoamericanas en Ciencia y Salud, electrónico, de 2002 a actualidad; Índice Artemisa-LILACS-BIREME, OPS, electrónico, 2005; Index Copernicus International, de 2006 a 2008.

\section{BOLETÍN INFORMATIVO. AHORA E-BOLETÍN}

Durante el bienio 1993-1994, en la transición de la directiva conducida por el Dr. Gilberto López Betancourt y el Dr. Lorenzo de la Garza 
(Figura 11), se fundó el Boletín informativo, que surgió como respuesta a la necesidad de establecer un vínculo más estrecho entre el asociado y la mesa directiva. A través de él, ésta informaría trimestralmente lo realizado en forma cotidiana por los comités que apoyan la labor del presidente.

Durante 19 años el boletín se publicó puntualmente cada trimestre en su versión impresa, y desde 2009-2010 en su versión electrónica.

\section{TRATADO DE CIRUGÍA GENERAL, 1. ${ }^{A}$ EDICIÓN $^{4}$ (FIGURA 12)}

En el verano de 1999, acompañamos al Dr. Fermín Martínez de Jesús, cirujano general del Hospital de Especialidades del Centro Médico Nacional del IMSS en la ciudad de Veracruz, a visitar al Ing. Alejandro Bravo Valdez, editor de McGraw-Hill, en el norte de la Ciudad de México. El Dr. Martínez estaba por publicar un libro con esta empresa y el motivo de la reunión era seleccionar la portada de la que sería la primera edición del libro Pie diabético.

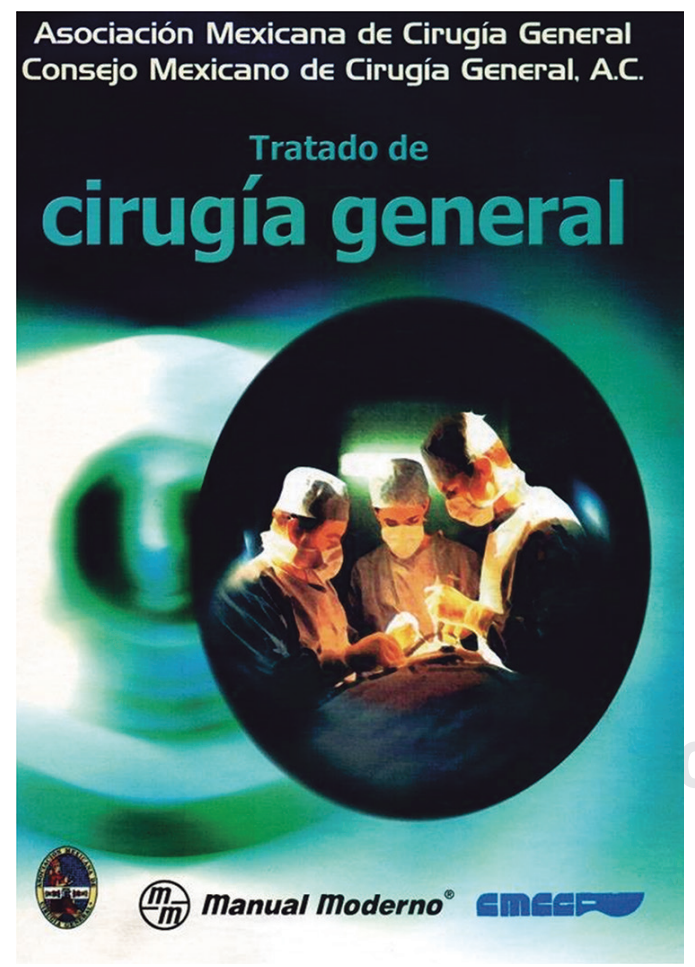

Figura 12. Tratado de cirugía general, 1. ${ }^{a}$ edición, 2003. atención integral. Mientras ellos charlaban observamos una hilera de textos sobre medicina; uno de ellos llamó nuestra atención por dos razones: el título: El internista y el grosor del lomo. Gentilmente, el Ing. Bravo, una persona de trato fácil y agradable, nos permitió tomarlo y hojearlo; al hacerlo, nos percatamos de que era un tratado de medicina interna escrito por internistas mexicanos y bajo la tutela editorial del Dr. Alberto Lifshitz, alguien de los que asistían a la reunión comentó que podríamos interesar a la mesa directiva de la asociación y sugerir la edición de una obra hecha por y para cirujanos generales.

Así ocurrió, y el Ing. Alejandro Bravo, con su peculiar "olfato" de editor, vislumbró que la obra se podría realizar. Gestionó una reunión con el presidente en turno y se firmó un convenio entre la asociación y la editorial McGrawHill, mediante el cual el financiamiento sería compartido entre la editorial y la asociación. Entusiasmados con la idea, se integró un comité editorial por parte de la asociación, se acordó que la obra sería coeditada por la asociación y el Consejo Mexicano de Cirugía General (CMCG), a quienes correspondería la figura autoral. Se diseñó el contenido del libro y, por su extensión, se acordó que llevaría el título de Tratado de cirugía general; deberían participar únicamente cirujanos generales, preferentemente de todo el país y asociados a la AMCG.

Sin embargo, ocurrieron varias situaciones que entorpecieron el buen camino del tratado: McGraw-Hill envió al Ing. Bravo a su oficina en Bogotá, Colombia, a solucionar algún problema editorial; su reemplazo mostró poco interés en la obra; no recibíamos las colaboraciones solicitadas, había retraso de meses, y McGraw-Hill pidió un adelanto monetario que a los directivos de la asociación les pareció exorbitante. Parecía que la obra se cancelaría; sin embargo, merced a los buenos oficios del Dr. Jesús Tapia y su amistad con el Dr. Jorge Aldrete Velasco, director de la editorial El Manual Moderno, quien se interesó por la obra y, después de algunos otros tropiezos, en el año 2003 se publicó el Tratado de cirugía general (Figura 12), 1. ${ }^{\text {a }}$ edición, ${ }^{4}$ cuando el presidente de la asociación era el Dr. Rafael Aguirre Rivero y el presidente del Consejo Mexicano de Cirugía General, el Dr. Lorenzo de la Garza Villaseñor. 
No es tema de esta crónica realizar un análisis exhaustivo del contenido de la obra, pero sí una revisión breve de algunos datos estadísticos que nos permitan evaluar la magnitud del esfuerzo realizado por los cirujanos generales mexicanos. Esta obra contó con 1,607 páginas, 191 capítulos, 191 autores y 73 coautores. El número de mujeres cirujanas que participaron en la edición del tratado fue de 18 (9.4\%); el número de autores que escribieron un capítulo fue de 134 (70.2\%), con un coautor: 44 (23\%), con dos coautores: $10(5.2 \%)$, y con tres coautores, $1.6 \%$. Asimismo, revisamos y cuantificamos la entidad federativa de donde son originarios los autores: el 86.3\% (167 de 196) pertenecen a cinco entidades federativas (DF, Jalisco, Nuevo León, Querétaro y Veracruz), el 13.7\% (29 autores) restante se agrupó en 10 entidades federativas. Una desviación observada consistió en que no logramos identificar el sitio de donde provenían ocho autores, pues no se mencionaba en la lista de colaboradores ni en los créditos de los capítulos. Tres autores residían en los EUA (Tucson, Texas y Cleveland respectivamente). Un dato positivo fue que el exagerado centralismo que existía en la edición de este tipo de obras dejó de ser preponderante. Si excluimos los autores no identificados y los de Estados Unidos, fueron 16 entidades federativas las que participaron en la creación del tratado. Los responsables de la coordinación editorial fueron el Dr. Rafael Aguirre Rivero y el Dr. Lorenzo De la Garza Villaseñor, presidentes en función de la AMCG y el CMCG, y los cirujanos Luis
Mauricio Hurtado López, José Marín Méndez, Carlos Melgoza Ortiz, Erick Romero Arredondo y Jesús Tapia Jurado; el coordinador general fue el Dr. Guillermo León López. (editor de Cirujano General).

Finalmente, la figura 13 muestra las partes que integran el tratado, 11 en total, el número de capítulos que contiene cada una de ellas y los cirujanos responsables de cada parte.

El éxito académico y comercial de la obra resultó excelente. Cinco años después, la misma editorial, El Manual Moderno, solicitó producir la segunda edición.

\section{TRATADO DE CIRUGÍA GENERAL, 2. ${ }^{A}$ EDICIÓN (2008) ${ }^{5}$ (FIGURA 14)}

La portada prácticamente es la misma que la de la primera edición, con algunos ajustes en los colores. Realizamos el mismo análisis cuantitativo al contenido de esta segunda edición y la comparamos con el obtenido en la primera. Los resultados son los siguientes: el número de páginas fue de 1,581, 26 menos que en la primera edición; en cambio se agregó un capítulo, que corresponde al último de la obra y trata un tema no publicado en la primera edición: "Bioética y cirugía en el siglo XXI".

En otros rubros, encontramos que el número de capítulos con un solo autor disminuyó de $113(59.1 \%)$ a 57 (29.6\%); en contrapartida, el número de coautores pasó de 78 (40.9\%) a 135 (70.4\%), principalmente en aquellos capítulos con dos coautores, que aumentó de tres (3.8\%) a $83(61.6 \%)$.

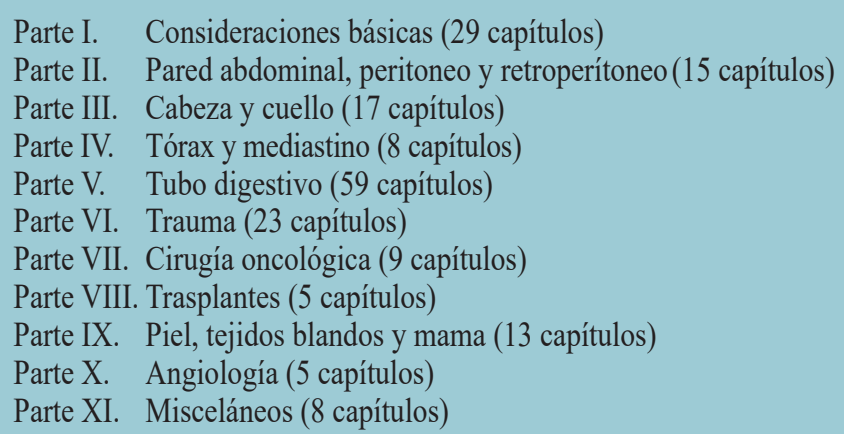

Dr. Alejandro González Ojeda (Jalisco)

Dr. Alberto Chousleb Kalach (México, DF)

Dr. Erich OP Basurto Kuba (México, DF)

Dr. Rafael Aguirre Rivero (Guerrero)

Dr. Gilberto López Betancourt (Nuevo León)

Dr. César Gutiérrez Samperio (Querétaro)

Dr. Francisco Cardoza Macías (B. California)

Dr. Luis Sigler Morales

Dr. Fermín Martínez de Jesús (Veracruz)

Dr. Fermín Martínez de Jesús (Veracruz)

Dr. Alejandro González Ojeda (Jalisco)

Figura 13. Contenido del Tratado de cirugía general, 1.a ed. 2003. 


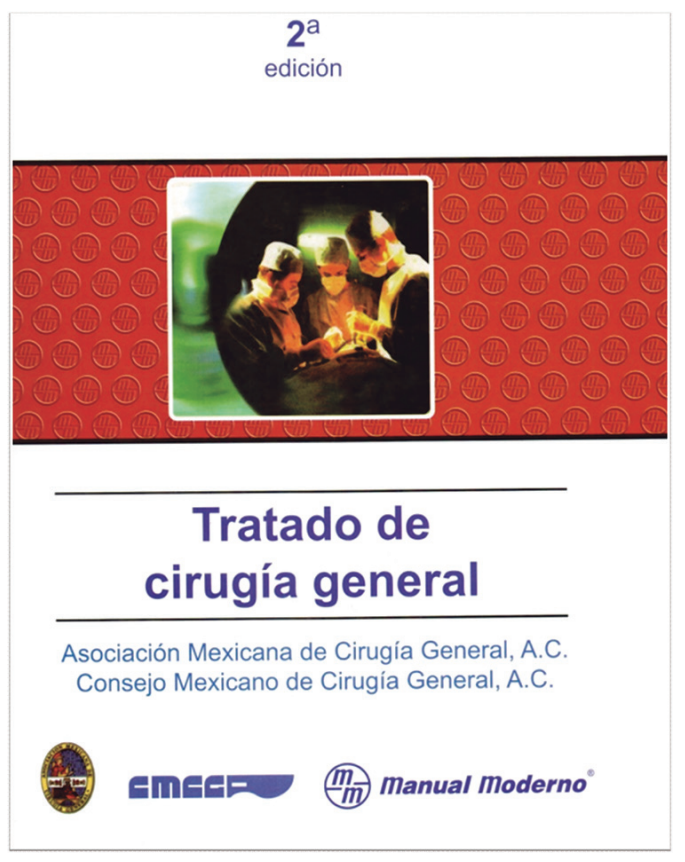

Figura 14. Tratado de cirugía general, 2. ${ }^{a}$ edición 2008.

El número de entidades federativas que se incorporaron en la segunda edición fue de cuatro (Coahuila, Estado de México, Durango y Chihuahua), y únicamente una entidad de la primera edición no participó en la segunda, Oaxaca. El número de autores aumentó a 274 (28\%).

Cuatro de las cinco entidades que participaron con mayor número de autores conservaron su posición y el número de autores de quienes no se pudo identificar su procedencia aumentó de ocho a 16.

De los ocho cirujanos que participaron como editores, cuatro procedían de la Ciudad de México, Ignacio J. Magaña Sánchez, José A. Carrasco Rojas, Guillermo León López y José A. Marín Méndez, y uno de cada una de las siguientes entidades: Coahuila (Roberto Bernal Gómez), Guanajuato (Éctor Jaime Ramírez Barba), Querétaro (Jesús A. Vega Malagón) y Veracruz (Alfonso G. Pérez Morales). El comité editorial tuvo 15 miembros, 11 de México, DF, los doctores Amado de Jesús Athié Athié, Ernesto A. Ayala López, David A. Banderas Tarabay, Francisco Campos Campos, Lilia Cote Estrada, Angélica H. González Muñoz, Luis A. Hernández Higareda, Luis M. Hurtado López,
Héctor F. Noyola Villalobos, Jesús Tapia Jurado y María G. Zermeño Gómez; tres de Querétaro, los doctores Enrique López Arvizu, Juan A. Castellanos Malo y César Gutiérrez Samperio; y uno de Guerrero, el Dr. Rafael Aguirre Rivero.

En 2016, se publicará la tercera edición del Tratado de cirugía general, que constará de 263 capítulos en dos tomos, con un enfoque programado hacia las competencias profesionales del cirujano con aplicación en el pre- $y$ postgrado, e incluirá la versión electrónica en disco compacto.

\section{HISTORIA DE LA CIRUGÍA GENERALENLAREPÚBLICAMEXICANA}

En el año 2008, cuando iniciaba su periodo en la presidencia de la Asociación, la Dra. Lilia Cote Estrada nos propuso un proyecto editorial sumamente interesante, que consistía en rescatar la historia de la cirugía general en cada uno de los estados de la república que conforman la asociación y la federación de colegios, y publicar uno o varios libros que la describieran; ello nos daría cohesión como federación y fortalecería a la asociación y a los colegios de cirugía general. La primera acción de la Dra. Cote, después de comprometernos a coordinar el proyecto, consistió en explicar la idea a los presidentes de los colegios de ese entonces, con una invitación para que cada uno de ellos designara en su colegio a un asociado, o varios, para investigar sobre el desarrollo de la cirugía general en su estado. Por nuestra parte, diseñamos un documento que denominamos "protocolo de trabajo", con guías e indicaciones generales al respecto; recomendábamos consultar a historiadores de su lugar de origen, como el cronista del estado, profesores de historia de la medicina en la escuela o facultad de medicina de su estado, si la hubiere, y desde luego, recurrir a textos de historia local y archivos para obtener información. Llenadas estas formalidades, algunos de los presidentes de colegios se interesaron en la idea y "empujaron" el proyecto. Tres años después, en 2011, se logró concretar y se editó el primer volumen de la serie (Figura 15). ${ }^{6}$

Este volumen contiene la historia de la cirugía general en los estados de Chihuahua, Tamaulipas, Nayarit, Querétaro, Quintana Roo y Chiapas, y de un hospital de la Cd. de México, 


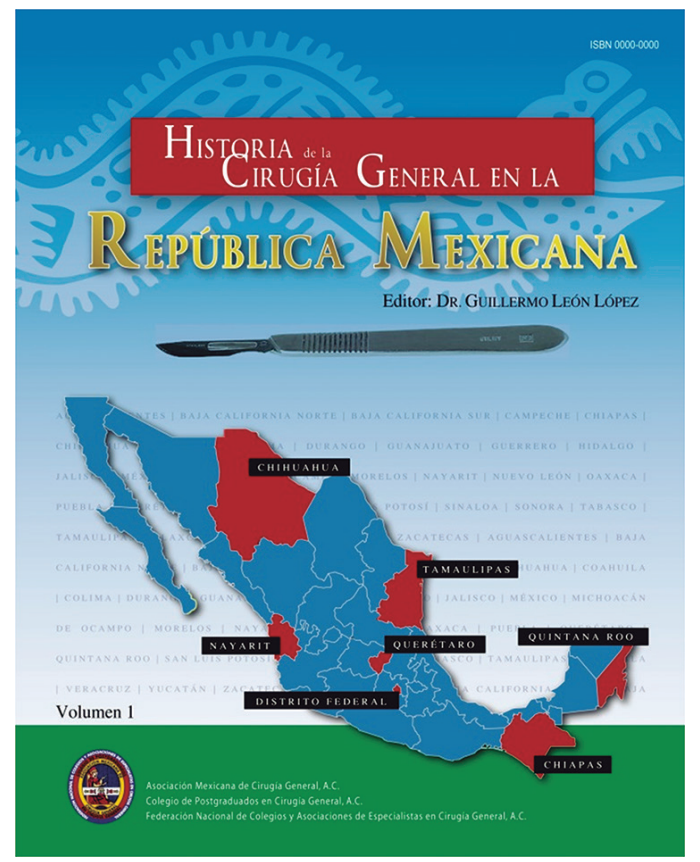

Figura 15. Portada del volumen I de Historia de la Cirugía General en la República Mexicana.

el Hospital General del Centro Médico Nacional "La Raza"; en la figura 16 se mencionan los autores de cada uno de esos capítulos.

En 2012 se editó el volumen II de la serie, ${ }^{7}$ (Figura 17) el cual contiene la crónica de la cirugía general en los estados de Sonora, Nuevo León y Aguascalientes y de cinco hospitales de la Cd. de México, DF: el Instituto Nacional de Ciencias Médica y Nutrición "Dr. Salvador Zubirán", el Hospital General de la Secretaría de Salud, el Hospital Central Militar, el antiguo Hospital Colonia de los FFCC y el Hospital General del Centro Médico Nacional del IMSS. En la figura 18 observamos el contenido y autores del volumen. Un año después, se publicó el volumen III (Figura 19), ${ }^{8}$ en él participaron los siguientes estados: Durango, Coahuila, San Luis Potosí, Jalisco y Puebla, y los siguientes hospitales: Hospital Central de Concentración Nacional de Petróleos Mexicanos (con sede en la Cd. de México, DF) y el Hospital Regional de Petróleos Mexicanos de Ciudad Madero, Tamaulipas. Los autores de esos capítulos se mencionan en la figura 20.

Cinco años después de planteado el proyecto, 33 cirujanos generales de 14 estados de
Índice

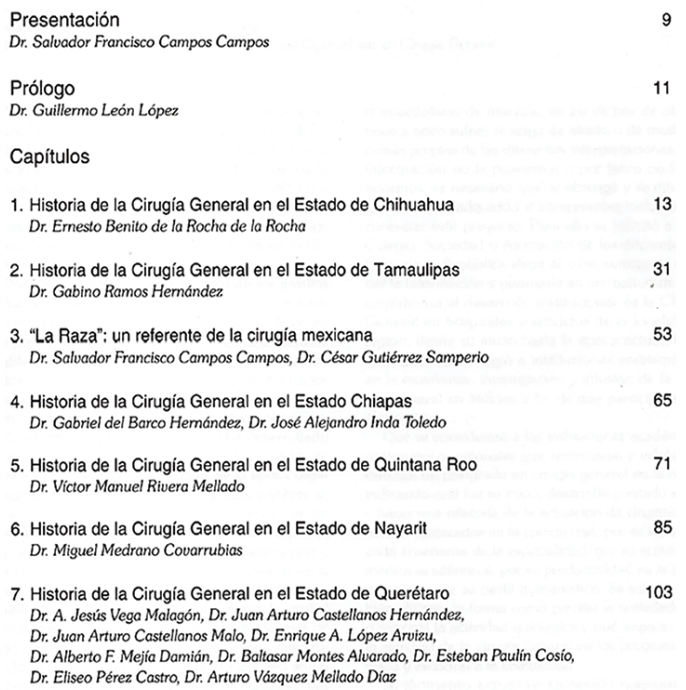

Figura 16. Autores y capitulos del volumen I de Historia de la cirugía general en la República Mexicana.

la república, sin dedicarse profesionalmente a escribir historia, han redactado la de la cirugía general en sus respectivas entidades; y 16 cirujanos generales de la Ciudad de México, DF escribieron la historia de la cirugía general en sus respectivos hospitales.

\section{PERFILES: CIRUJANOS GENERALESMEXICANOS DELSIGLOXX ${ }^{9}$ (FIGURA 21)}

Entre los años 2002 y 2005 se publicaron en el Boletín informativo, en ese entonces únicamente en versión en papel, varios textos con el título de "Perfiles", en los que se describía, como en una fotografía "de perfil", la actividad quirúrgica de algunos cirujanos generales mexicanos durante el siglo XX, en sus aspectos quirúrgico, académico, docente y profesional; todos eran originarios de algún estado de la República Mexicana y la Ciudad de México, y habían, preferentemente, aunque no de forma obligatoria, pertenecido a la AMCG, considerando que la fecha de fundación de ésta fue en el año 1974. No se trataba de escribir una biografía del personaje, sino únicamente los rasgos más 


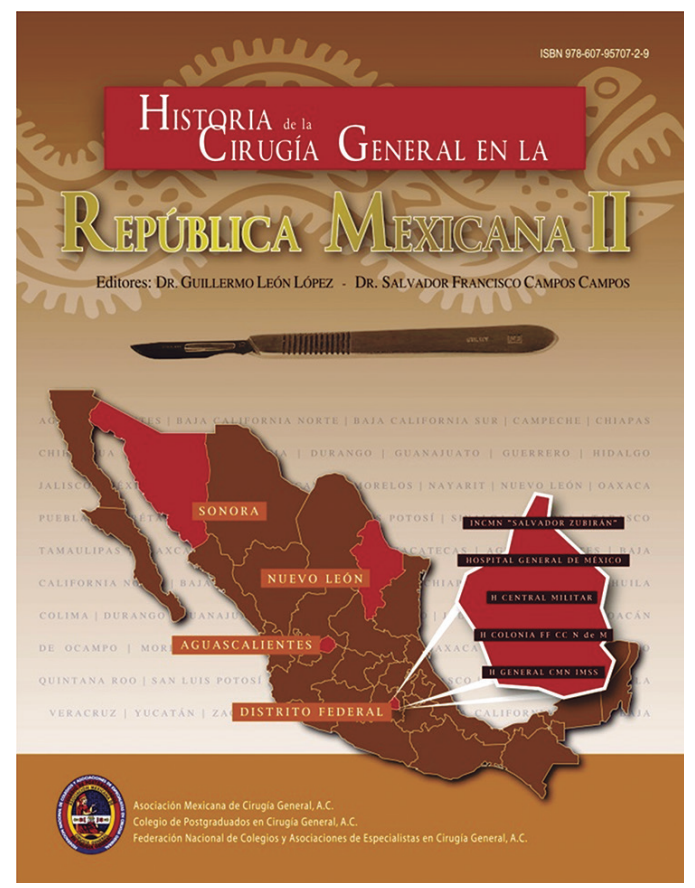

Figura 17. Portada del volumen II de Historia de la Cirugía General en la República Mexicana.

\section{ÍNDICE}

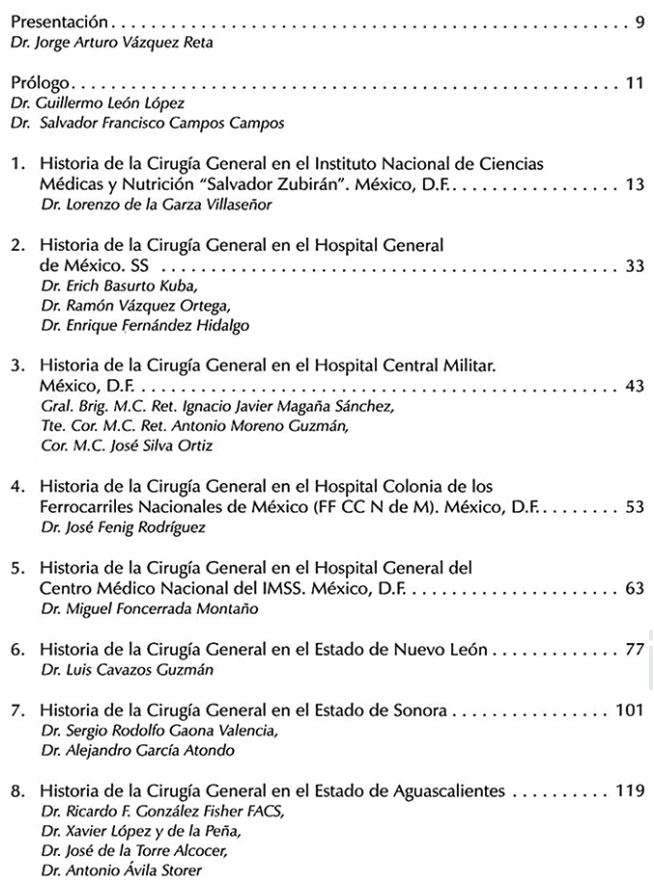

3. Historia de la Cirugía General en el Hospital Central Militar. México, D.F................................ 43 Gral. Brig. M.C. Ret. Ignacio Javier Magaña Sánchez, Tte. Cor. M.C. Ret. Antonio Moreno Guzmán. Cor. M.C. José Silva Ortiz

4. Historia de la Cirugía General en el Hospital Colonia de los Ferrocarriles Nacionales de México (FF CC N de M). México, D.F. . . . . . 53 Dr. José Fenig Rodriguez

5. Historia de la Cirugía General en el Hospital General del Centro Médico Nacional del IMSS. México, D.F. . . . . . . . . . . . 63 Dr. Miguel Foncerrada Montanio

6. Historia de la Cirugía General en el Estado de Nuevo León.......... 77 Dr. Luis Cavazos Guzmán

7. Historia de la Cirugía General en el Estado de Sonora ............ 101 Dr. Sergio Rodolfo Gaona Valencia Dr. Alejandro García Atondo

8. Historia de la Cirugía General en el Estado de Aguascalientes ......... 119 Dr. Ricardo F. González Fisher FACS, Dr. Xavier topez y de la Peria Dr. Antonio Ávila Storer

Figura 18. Autores y capítulos del volumen II de Historia de la Cirugía General en la República Mexicana.

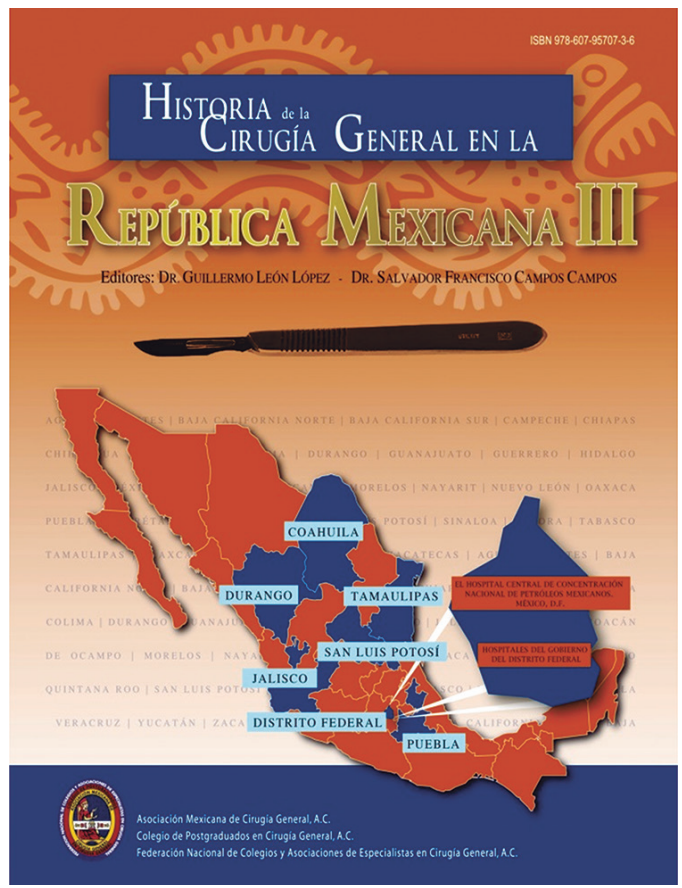

Figura 19. Portada del volumen III de Historia de la Cirugía General en la República Mexicana.

\section{ÍNDICE}

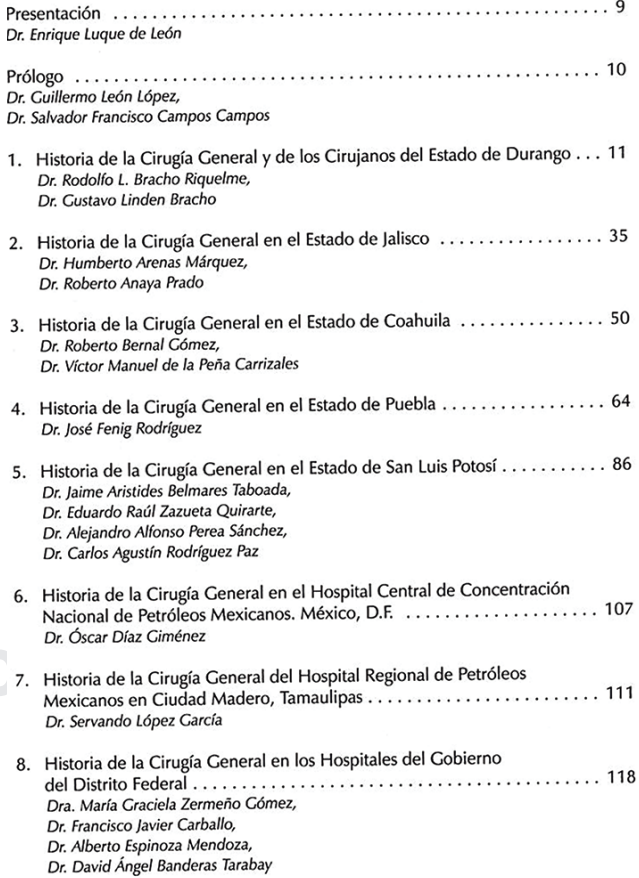

5. Historia de la Cirugía Ceneral en el Estado de San Luis Potosí . ........ 86 Dr. Jaime Aristides Belmares Taboada, Dr. Eduardo Raúl Zazueta Quirarte, Dr. Alejandro Alfonso Perea Sánchez Dr. Carlos Agustín Rodríguez Paz

6. Historia de la Cirugía General en el Hospital Central de Concentración Nacional de Petróleos Mexicanos. México, D.F. ............... 107 Dr. Óscar Díaz Ciménez

7. Historia de la Cirugia General del Hospital Regional de Petróleos Mexicanos en Ciudad Madero, Tamaulipas ................... 111 Dr. Servando López Carcía

8. Historia de la Cirugía Ceneral en los Hospitales del Cobierno del Distrito Federal ................ Dr. Francisco Javier Carballo, Dr. Alberto Espinoza Mendoza,

Figura 20. Autores y capitulos del volumen III de Historia de la Cirugía General en la República Mexicana. 


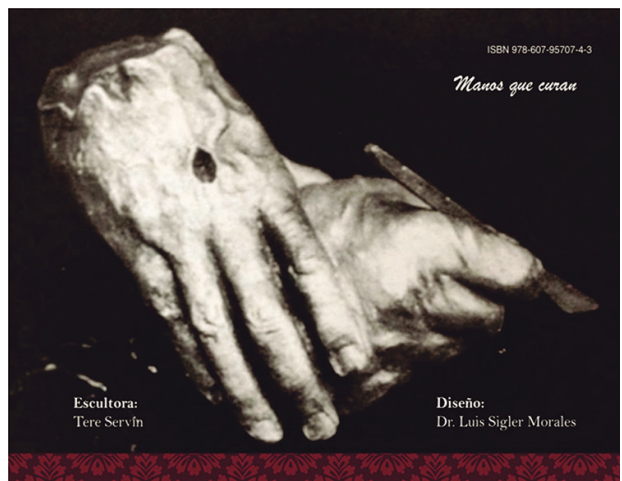

\section{Perfiles:}

Cirujanos Generales Mexicanos

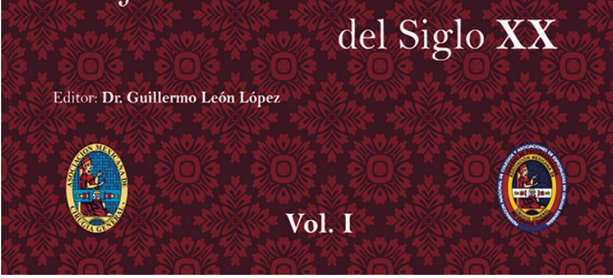

Figura 21. Portada del libro Perfiles.

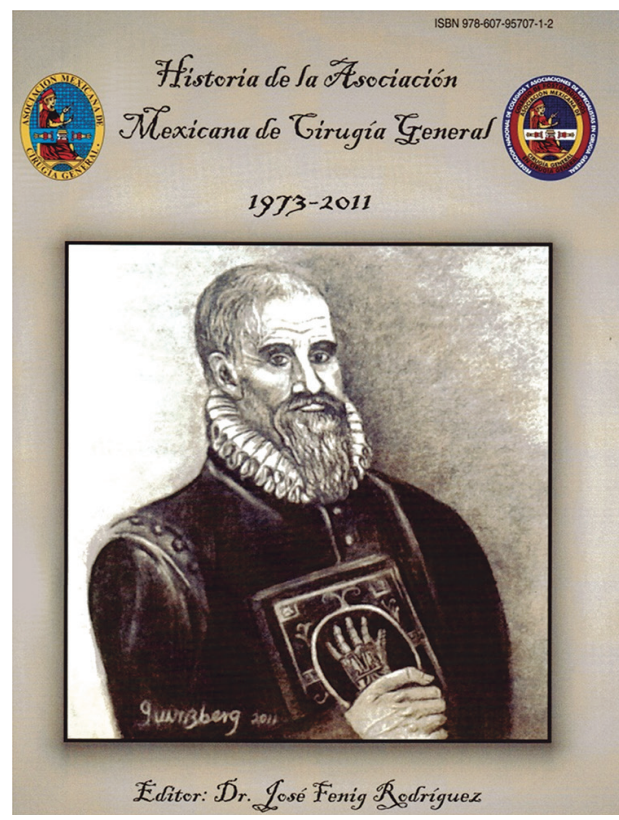

Figura 22. Portada del libro Historia de la Asociación Mexicana de Cirugía General.
1. "Código de Bioética". 1996. Autores: Drs. Leonel Barrera Cantú, Miguel F. Ayala Fuentes, Jorge Bautista O'. Farril, Alejandro Híjar Godínez y Adolfo Martínez Licona.

2. "Maestros de la Cirugía Moderna" 1996. Autor: Dr. Luis H. Toledo-Pereyra. Derechos Reservados 1996 Asociación Mexicana de Cirugía General. Derechos Reservados 1996 Fondo de Cultura Económica. ISBN 968-16-5065-4 P.121

3. "Historia, Cirugía y Cultura". 1998. Autor: Dr. Luis H. Toledo-Pereyra. Derechos Reservados 1998 Asociación Mexicana de Cirugía General. Derechos Reservados 1998 J.G.H. Editores, S. A de C. V. ISBN 968-7860-78-2 P.174

4. "Discurso a los Cirujanos". 1998. Autor: Paul Valery. Traducción y prólogo: Dr. Francisco González Crussi. Ed. Verdehalago. ISBN 968-6767-79-7

5. "Maestros de la Cirugía Contemporánea”. 1999. Autor. Dr. Luis H. Toledo-Pereyra. 1ª Edición 1999 Derechos Reservados, 1999. ISBN 970-681-029-3, P.132

6. "Maestros Nobel de la Cirugía". 2000. Autores: Dr. Luis H. Toledo P., Dr. Gustavo Martínez Mier. 1a. Edición 2000. Derechos Reservados 2000 J.G.H. Editores. ISBN 970-681-055-2, P.148

7. "Iatrogenia en Cirugía General". 2001. Autores: Dr. V. M. Arrubarrena Aragón, Dr. César Gutiérrez Samperio. Edición Asociación mexicana de Cirugía General, A. C. (Sin ISBN)

8. "Código de ética". 2002. Autores: Dr. V. M. Arrubarrena A, Dr. César Gutiérrez Samperio, Dr. Jorge Bautista O' Farril. Editorial El Manual Moderno. ISBN 970-729-000-5, P.46

9. "Cirugía en el paciente geriátrico". 2006. Autores: Dra. Lilia Cote Estrada, Dr. David Olvera Pérez. Editorial Alfil, S. A de C. V. ISBN 968-7620-54-4 P. 769

10. "Código de ética" 2009. C.P.C.G. y A.M.C.G. Comité de ética. Autores: Dr. César Gutiérrez Samperio, Dr. V.M. Arrubarrena Aragón y Dr. Federico Sandoval Olvera. Edición Asociación mexicana de Cirugía General, A. C. (Sin ISBN)

11. "La cirugía General y el Cirujano". 2011. Editor: Dr. Jesús Vega Malagón. Derechos Reservados Universidad Autónoma de Querétaro. ISBN 978-607-7740-60-5, P.541

12. "Innovación y Descubrimientos en Cirugía, Historia y Entorno Humanístico" 2013. Autor: Dr. Luis H. Toledo-Pereyra. (Traducción) Copyright 2013 para la edición en español, Landes Bioscience. ISBN 978-607-95707-5-0, P.139. Impreso en México por Graphimedic, S. A de C. V.

\section{Figura 23.}

Obras relacionadas con la historia de la cirugía avaladas por la Asociación Mexicana de Cirugía General. 
distintivos de su actividad como cirujano general, aun cuando también se debía considerar que la especialidad tiene su punto de partida fundacional en la fecha en que se estableció el Consejo Mexicano de Cirugía General.

Con esta idea editamos una veintena de "Perfiles" en el Boletín informativo de la asociación, con cirujanos generales que cumplían con los criterios de selección señalados. Producto de esos textos es la publicación que se editó en noviembre de 2013 bajo el título de Perfiles: Cirujanos Generales Mexicanos del Siglo XX. ${ }^{9}$

\section{HISTORIA DE LA ASOCIACIÓN MEXICANA DE CIRUGÍA GENERAL ${ }^{3}$ (FIGURA 22)}

En 2011 se publicó, bajo la coordinación editorial del Dr. José Fenig Rodríguez, expresidente de la AMCG (1989-1990), el libro Historia de la Asociación Mexicana de Cirugía General, cuyo contenido agrupa los discursos de clausura de la gestión de cada uno de los, hasta esa fecha, 32 expresidentes de la asociación.

Para concluir este breve repaso de los 40 años de actividad editorial, sólo mencionaremos los títulos de otros libros que han sido patrocinados por la Asociación Mexicana de Cirugía General, así como los nombres de sus autores y sus años de publicación (Figura 23).

\section{REFERENCIAS}

1. Asociación Mexicana de Cirugía General, AC. Estatutos 2013. Artículo 2. Del objeto. Inciso H. pp. 42-43 (Fecha de consulta: marzo de 2016.)
2. Rodríguez MS. Algunos hechos no conocidos de la fundación de la Asociación Mexicana de Cirugía General. Cir Gen. 2007; 29: 297-300.

3. Fenig RJ. Historia de la Asociación Mexicana de Cirugía General. 1973-2011. México: Ed. Graphimedic; 2011. pp. 43-46. ISBN: 978-607-95707-1-2.

4. Asociación Mexicana de Cirugía General. Consejo Mexicano de Cirugía General. Tratado de Cirugía General. México, DF: El Manual Moderno, SA de CV; 2003. 1620 pp. ISBN: 968-426-990-0.

5. Asociación Mexicana de Cirugía General. Consejo Mexicano de Cirugía General. Tratado de Cirugía General. 2. ${ }^{a}$ edición. México, DF: El Manual Moderno, SA de CV; 2008. 1580 pp. ISBN: 978-970-729-368-7.

6. Asociación Mexicana de Cirugía General. Historia de la cirugía general en la República Mexicana. Vol. I. Estados: Chihuahua, Chiapas, Distrito Federal, Nayarit, Querétaro, Quintana Roo, Tamaulipas. México: Ed. Graphimedic; 2011. 121 pp. ISBN: 978-607-95707-0-5.

7. Asociación Mexicana de Cirugía General. Historia de la cirugía general en la República Mexicana. Vol. II. Estados: Sonora, Nuevo León, Aguascalientes, Distrito Federal: INCMN "Salvador Zubirán", Hospital General de México, H. Central Militar, H. Colonia FFCC N de M, H General CMN IMSS. México: Ed. Graphimedic; 2012. 133 pp. ISBN: 978-607-95707-2-9.

8. Asociación Mexicana de Cirugía General. Historia de la cirugía general en la República Mexicana. Vol. III. Estados: Coahuila, Durango, Jalisco, Tamaulipas, San Luis Potosí, Puebla y Distrito Federal: Hospitales del Gobierno del Distrito Federal, Hospital Central de Concentración Nacional de Petróleos Mexicanos. México: Ed. Graphimedic; 2013. 128 pp. ISBN: 978607-95707-3-6.

9. Asociación Mexicana de Cirugía General. Perfiles: cirujanos generales mexicanos del siglo XX. México: Ed. Graphimedic; 2013. 183 pp. ISBN: 978-60795707-4-3.

Correspondencia:

Dr. Guillermo León López

Ingenieros Civiles Núm. 62

Col. Jardines de Churubusco 09410

Del. Iztapalapa, Ciudad de México.

E-mail: doctorleon@prodigy.net.mx 